\title{
Central systolic blood pressure (cSBP) to brachial systolic blood pressure (brachSBP) ratio reproducibility during antihypertensive therapy
}

\author{
Bulas $\mathrm{J}^{1}$, Potocarova $\mathrm{M}^{1}$, Murin $\mathrm{J}^{1}$, Wimmerova $\mathrm{S}^{3}$, Gaspar $\mathrm{L}^{1,4}$, Turecky $\mathrm{L}^{5}$, \\ Kovacova $\mathrm{E}^{1}$, Kupcova $\mathrm{V}^{2}$
}

Ist Department of Internal Medicine, Faculty of Medicine, Bratislava, Slovakia. jozef2bulas@gmail.com

\begin{abstract}
OBJECTIVES: Higher CSBP than brachial SBP in individual patient increases cardiovascular (CV) risk. For follow-up it is important to assess the reproducibility of such measurements. The aim of this study was to assess the reproducibility of these differences, expressed as a CSBP/BrachSBP ratios.

SUBJECTS AND METHODS: Eighty-three patients on antihypertensive therapy were analysed for the reproducibility of such ratios after time interval of several month up to several years. For CSBP estimation, we used the Arteriograph (Tensiomed Ltd.), based on blood pressure measurements by cuff on oscillometric principle, using pulse wave analysis (PWA) for assessment of CSBP.

RESULTS: The proportion of patients retained the same characteristics (either higher central or higher peripheral SBP) between the first and second measurement was $71.1 \%$. The association between 1 st and 2nd measurement, was statistically significant, $p<0.001$.

CONCLUSION: In our study, a high proportion (60\%) of treated hypertensive patients had CSBP higher than brachial SBP, which may adversely influence their prognosis. This characteristic is highly reproducible. Taking into the account these differences may increase the exactness of CV risk estimation and may contribute to explanation of residual risk of individual patient (Tab. 3, Fig. 1, Ref. 28). Text in PDF www.elis.sk KEY WORDS: central systolic blood pressure, central systolic to brachial systolic ratio reproducibility, arterial hypertension.
\end{abstract}

\section{Introduction}

Arterial hypertension belongs to the most important cardiovascular (CV) risk factors. Classical blood pressure (BP) measurements on the arm over the brachial artery (auscultatory or oscillometric) do not allow to assess the blood pressure in the central arterial compartment, especially in the ascending aorta (1). The left ventricular load is more exactly determined by the central systolic blood pressure (cSBP) than by brachial systolic

${ }^{1}$ Ist Department of Internal Medicine, Faculty of Medicine and University Hospital, Comenius University, Bratislava, Slovakia, ${ }^{2}$ IIIrd Department of Internal Medicine, Faculty of Medicine, Comenius University, Bratislava, Slovakia, ${ }^{3}$ Slovak Medical University, Faculty of Public Health, Bratislava, Slovakia, ${ }^{4}$ Faculty of Health Sciences, University of Ss Cyril and Methodius in Trnava, Slovakia, and ${ }^{5}$ Institute of Medical Chemistry, Biochemistry and Clinical Biochemistry, Faculty of Medicine, Comenius University, Bratislava, Slovakia

Address for correspondence: J. Bulas, Assoc Prof, MD, PhD, Ist Department of Internal Medicine, Faculty of Medicine, Mickiewiczova 13, SK-813 69 Bratislava, Slovakia.

Phone: +421.2 .57290249$

Acknowledgement: This research work was supported by the scientific grants VEGA 1/0826/18 and VEGA 1/0807/18 of the Slovak Ministry of Education. blood pressure (brachSBP), and according to many clinical studies, cSBP is more important prognostic factor than brachial systolic blood pressure (2).

There are several measurement techniques enabling non-invasive assessment of central systolic blood pressure. Most of them require a highly trained operator and special equipment (3).

In recent years, new devices for central systolic blood pressure measurement emerged on the market; they are easy to use and not requiring highly trained operator. There are based on Pulse Wave Analysis (PWA) approach, pressure wave is detected oscillometrically by blood pressure cuff over the brachial artery $(4,5)$.

From many previous studies it is known, that the pulsatile character of blood flow in arteries determines the systolic blood pressure throughout all the arterial tree. It is generally accepted that diastolic blood pressure is roughly the same in all arteries and the pressure wave amplitude defines the systolic blood pressure in each point of arteries. Important organs such as: heart, brain and kidneys are directly exposed to systolic pulsatory flow, which in hypertension, may be deleterious to vasculature. Therefore, the scientific and clinical attention is oriented to systolic blood pressure and to analysis of blood pressure waves $(1,6)$. Despite the traditionally presented position that brachSBP should be due to peripheral amplification always higher than cSBP, the results of 
several papers however suggest, that the values of cSBP may be higher than brachSBP in the patients with stiffer arteries $(7,8)$.

Kim et al (9) in the group of 335 patients at a very high risk of atherosclerosis calculated the difference between the Peripheral and Central systolic blood pressure (P-CSBP), and according to the results, divided the patients into $\mathrm{P}-\mathrm{CBP}$ positive and $\mathrm{P}-\mathrm{CBP}$ negative. During the median follow-up of 12.6 months, the negative difference $\geq-8 \mathrm{mmHg}$ (i.e. higher cSBP) was associated with a 3-fold higher likelihood of poor prognosis. On the other hand, Ryuzaki and al (10), used the $\Delta$ (delta) values for the differentiation of patients according to the prevailing central, cSBP or brachial, brachSBP values. The $\Delta$ (delta) value was calculated as cSBP office brachSBP; the patients were divided into those with positive $\Delta$ values (the $\Delta+$ group) and those with negative $\Delta$ values (the $\Delta$ - group). Into the $\Delta^{+}$group belonged the patients with a higher central systolic (cSBP) than brachial systolic (brachSBP) blood pressure. Patients in the $\Delta+$ group had a significantly higher cSBP, increased augmentation indexes, negative correlation with eGF and endothelial dysfunction. They concluded that cSBP in their group of patients were mostly higher than brachSBP, which seemed contradictory to general opinion that brachSBP is higher than cSBP.

In our previous studies (11), we noticed that the values of central systolic blood pressure may differ from the brachial blood pressure values. Surprisingly, more than a half of our patients had a central systolic blood pressure higher than peripheral (brachial) blood pressure. This finding is in opposition to prevailing opinion, that brachial systolic blood pressure is always higher than the central one. This position can be elucidated by reasoning, that those conclusions are based on findings in younger or healthy persons in physiological state, with elastic arteries, when the brachial systolic blood pressure is higher because of peripheral BP amplification $(1,2,8)$ and also as the consequence of methodological differences (12). Another group of authors (13) reported that invasively measured cSBP and brachSBP in the patients with hypertension, older than 50 years, undergoing cineangiography, all had SBP in the root of aorta higher than blood pressure in radial artery. Also, the variability of central systolic blood pressure may influence the results, as was reported in Anglo-Cardiff Collaborative Trial II describing the large central systolic blood pressure variability increasing with age and overlapping values between a high normal and stage I hypertension (14).

\section{Objective of the study}

The aim of this work was to study the differences (or ratios) between the CSBP and brachSBP and to evaluate if these differences are reproducible after several month/years of treatment, on repeated measurements. For easier comparison of these two results obtained from every patient, we expressed these differences as Central to Brachial Systolic blood pressure ratio, which allows to compare these different measurements regardless of absolute blood pressure values or the differences. The resulting value higher than 1.0 meant that the central systolic blood pressure was higher than brachial one, and the resulting value below 1.0 meant that brachial systolic blood pressure was higher than central one.

\section{Subjects and methods}

Patients analysed were originally involved in the research project studying central haemodynamic and preclinical cardiovascular disease, which was approved by the Ethical committee of University Hospital. Patients signed the informed consent form after we explained to them the nature and aim of this type of non-invasive BP measurement. The patients undergone a basic clinical examination, most of them had a blood pressure well controlled, only some of them obviously needed an intensifying therapy. The exclusion criteria were: atrial fibrillation, advanced renal failure [glomerular filtration rate $-\mathrm{GFR}<60 \mathrm{ml} /(\mathrm{min} \cdot \mathrm{m} 2)$ ], and/or significantly increased liver function test values (liver function test values greater than 3 times the upper normal limit). The treatment of hypertension in the patients evaluated was based on antagonists of RAAS (inhibitors of angiotensin converting enzyme or Angiotensin II receptor antagonists) combined with calcium channel blockers, mainly amlodipine, with addition of low dose of metabolically neutral diuretic (indapamide).

The analysed group consisted of 83 patients, 36 males (43.4\%) and 47 females $(56.6 \%$ ), of the mean age $65.8 \pm 11.7$ years (males $63.2 \pm 13.5$ years, females $67.9 \pm 9.7$ years). In the whole group, the mean brachial systolic BP was $137 \pm 13.6 \mathrm{mmHg}$, and the mean cSBP was $137 \pm 16.2$ (Tab. 1).

The invasively and non-invasively validated instrument, Arteriograph Tensiomed Ltd (H-1103 Budapest, Hungary), was used to estimate the central BP (4).

It works based on the oscillometric principle, using one cuff both for BP measurement and waveform detection for pressure wave analysis (one measurement site, single point analysis) (15).

The cuff for BP and central haemodynamic evaluation was tightly fastened on the dominant arm above the elbow as recommended in the user's manual (16). The pressure wave is selfcalibrated using the brachial pressure value, which was obtained during the same measurement cycle (16). One measurement cycle lasts 2 to 3 minutes. Patients were examined after 5 to 10 minutes of rest in the supine position. After placing the cuff in the proper position on the arm, the actual measurement is automated and

Tab. 1. Basic Characteristic of the group of patients.

\begin{tabular}{|c|c|c|c|}
\hline \multirow{2}{*}{ Parameter } & \multicolumn{3}{|c|}{ Number } \\
\hline & total & Males & Females \\
\hline Number (\%) & $83(100 \%)$ & $36(43 \%)$ & $47(57 \%)$ \\
\hline Age (years) \pm SD & $65.8 \pm 11.7$ & $63.2 \pm 13.5$ & $67.9 \pm 9.7$ \\
\hline Height $(\mathrm{cm}) \pm \mathrm{SD}$ & $169.1 \pm 9.9$ & $177.1 \pm 8.0$ & $162.7 \pm 6.0$ \\
\hline Weight (kg) \pm SD & $81.9 \pm 15.9$ & $88.9 \pm 15.7$ & $76.2 \pm 13.8$ \\
\hline $\mathrm{BMI}\left(\mathrm{kg} / \mathrm{m}^{2}\right) \pm \mathrm{SD}$ & $28.5 \pm 4.7$ & $28.4 \pm 4.7$ & $28.7 \pm 4.7$ \\
\hline \multicolumn{4}{|l|}{ Mean BP } \\
\hline \multicolumn{4}{|l|}{ 1st measurement: } \\
\hline Brach-SBP $(\mathrm{mmHg}) \pm \mathrm{SD}$ & $137 \pm 13.6$ & $139 \pm 12.3$ & $136 \pm 16$ \\
\hline $\mathrm{CSBP}(\mathrm{mmHg}) \pm \mathrm{SD}$ & $137 \pm 13.6$ & $139 \pm 16.5$ & $138 \pm 16.0$ \\
\hline \multicolumn{4}{|l|}{ 2nd measurement: } \\
\hline Brach-SBP $(\mathrm{mmHg}) \pm \mathrm{SD}$ & $135 \pm 15.3$ & $133 \pm 13.2$ & $135 \pm 17.0$ \\
\hline $\mathrm{CSBP}(\mathrm{mmHg}) \pm \mathrm{SD}$ & $135 \pm 18.2$ & $133 \pm 17.2$ & $137 \pm 19.0$ \\
\hline
\end{tabular}


Tab. 2. Reproducibility of Central SBP to BrachSBP ratio between 1st and 2nd measurements.

\begin{tabular}{lll}
\hline $\begin{array}{l}\text { Central Systolic Blood Pressure to brachial Systolic BP ratio (mean } \pm \text { SD). } \\
\text { Comparison of ratios between 1st and 2nd measurements. }\end{array}$ \\
$\begin{array}{lll}\text { 1st measurement } & \text { CSBPao-1/ Brach-SBP-1 } & \begin{array}{l}0.9979 \pm 0.0626 \\
\text { Median }=1.029\end{array} \\
\hline \text { 2nd measurement } & \text { CSBPao-2 / Brach-SBP-2 } & \begin{array}{l}1.0013 \pm 0.05926 \\
\text { Median }=1.022\end{array}\end{array}$ NS ( $\left.=0.785\right)$ \\
\hline
\end{tabular}

Tab. 3. Association between 1st and 2nd measurements.

\begin{tabular}{|c|c|c|c|c|c|}
\hline & \multicolumn{2}{|c|}{1 st measurement } & \multirow{2}{*}{ Total } \\
\hline & & & Higher CSBP & Higher BrachSBP & \\
\hline \multirow{6}{*}{ 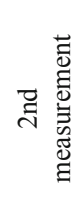 } & & No & 38 & 12 & 50 \\
\hline & Higher CSBP & $\%$ within 1 st measurement & $76.0 \%$ & $36.4 \%$ & \\
\hline & & $\%$ of Total & $45.8 \%$ & $14.5 \%$ & $60.2 \%$ \\
\hline & & No & 12 & 21 & 33 \\
\hline & Higner & $\%$ within 1 st measurement & $24.0 \%$ & $63.6 \%$ & 3980 \\
\hline & & $\%$ of Total & $14.5 \%$ & $25.3 \%$ & $39.8 \%$ \\
\hline \multirow{2}{*}{\multicolumn{2}{|c|}{ Total }} & No & 50 & 33 & 83 \\
\hline & & $\%$ of Total & $60.2 \%$ & $39.8 \%$ & $100.0 \%$ \\
\hline
\end{tabular}

That means that the probability that patient remains in the same hemodynamically characterised group also in the second measurement is 0.711 .

We can see that in each series of measurements, the higher values of cSBP were present more frequently (in $60.2 \%$ ), than the higher brachSBP (in $39.8 \%$ ).

If we evaluate the whole group of 83 patients from the aspect of repeatability of the cSBP/brachSBP ratio, we see, that from 50 patients with the centralSBP prevailing, 38 $(76 \%)$ remain in the same group, and from the group with brachSBP prevailing remain in the same group 21 (64\%) from 33 patients. That means that $71.1 \%$ (59 patients) from the whole group of 83 patients remain in the same position also in the second measurement. Of those, who had a higher central pressure in the first measurement, 12

operator-independent, and the results of measurements depend solely on the measuring device.

The Arteriograph provides several parameters of peripheral and central haemodynamic; for this analysis we used the values of central systolic blood pressure (cSBP) brachial systolic (brachSBP), diastolic pressure, pulse pressure (PP) and heart rate (HR).

\section{Statistical methods}

We used the mean, standard deviation and median to characterize the evaluated data. For statistical evaluation (analysis) we used independent two-sample T-test, non-parametric paired Wilcoxon test, Pearson Chi-squared test of independence and linear regression.

\section{Results}

There were no statistically significant differences in the mean blood pressure values among the subgroups of the patients, and in the mean values between central and peripheral BP.

When we evaluated the ratio of central systolic to peripheral systolic blood pressure (cSBPao/brach-SBP) we found that there were no significant differences between the ratios, obtained in the first measurement and in the second measurements. The mean values of ratios were calculated from the individual ratios of the central-SBP to brachial SBP. Statistical analysis using non-parametric Paired Wilcoxon test revealed the value of $p=0.785$ (Tab. 2).

The overview of hemodynamic type distribution: either with a higher central systolic blood pressure prevailing, or with a higher brachial systolic blood pressure prevailing, is presented in Table 3.

The proportion of patients, who did not change the characteristic position (either higher central or higher peripheral SBP) is given in main diagonal (from the left to right, framed by thick lines) is 59 , that is $71.1 \%$.

Using the Pearson Chi-squared test of independence we proved a statistically significant association between 1 st and 2 nd measurement, $\mathrm{p}<0.001$. patients moved into opposite group in the second measurement (i.e. $24 \%$ of 50 patients).

The grey boxes (with open circles) represent the proportion of those patients, who changed their previous hemodynamic position (migrated either from the higher central pressure group to the higher peripheral pressure group or vice versa).

White boxes (with black circles) represent the proportion of stable patients, who remained in the same hemodynamic group with the same SBPao / SyS ratio.

We demonstrated that in the group of patients in whom there was no transfer between groups, there was a statistically significant linear dependence of the cSBP / brachSBP ratio between the 1st

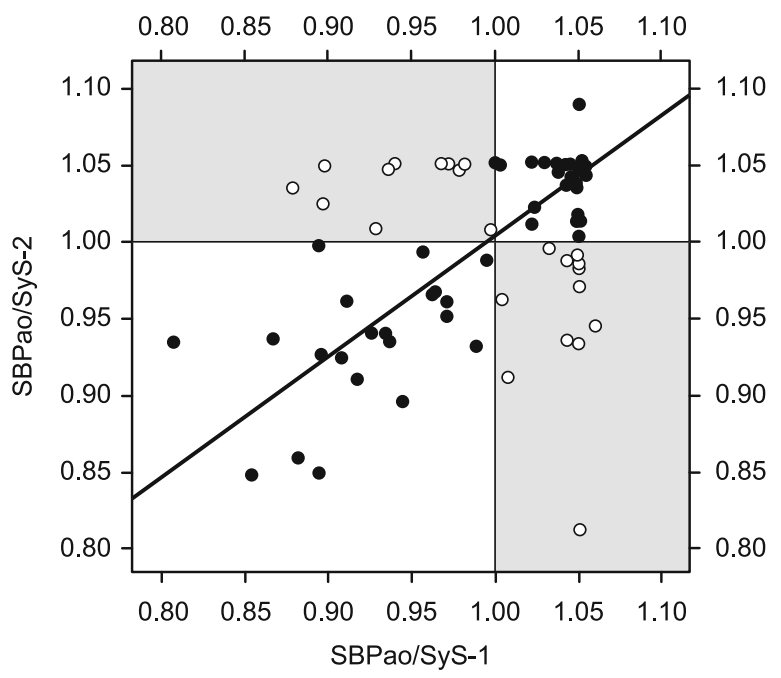

Fig. 1. Relationship between 1st (SPBAo/SyS-1) and second (SPBAo/ SyS-2) measurement. SBPao/SyS-1 = ratio of Central Systolic BP/ Brachial Systolic BP in the 1st measurement. SBPao/SyS-2 = ratio of Central Systolic BP/Brachial Systolic BP in the 2nd measurement. 
and 2nd measurements, regression coefficient $\beta$ (slope) $=0.797, \mathrm{p}$ $<0.001$, coefficient of determination, $\mathrm{R}^{2}=75.2 \%$ (Fig. 1).

The analysis showed that the proportion of patients with a higher central or higher peripheral systolic pressure did not differ significantly between the first and second measurements.

In the statistical analysis of parameters that could affect the migration of patients from one group to another (or to characterize them), we did not find the effect of gender, age, or the number of drugs used. Only BMI proved to be a significant factor for shifting patients between the groups; patients with statistically significantly higher BMI values migrated; patients, who migrated between the groups had significantly higher BMI values; independent twosample T-test: $p=0.012$. Patients, who did not change grouping according to the ratio of central to peripheral blood pressure had BMI of $27.7 \pm 4.3 \mathrm{~kg} / \mathrm{m}^{2}$ at the first measurement; patients, who changed classification according to pressure ratio had BMI of $30.5 \pm 4.9 \mathrm{~kg} / \mathrm{m}^{2}$.

\section{Discussion}

Central systolic blood pressure is in the centre of interest of researchers and clinicians for many years. Elastic central arterial compartment influences blood pressure, decreases the heart workload, dampens the pressure waves in central arterial compartment and in physiological state increases the efficacy of heart work and helps to change the pulsatory flow to more fluent flow in peripheral arterial tree $(17,18)$.

Values of cSBP measured or estimated may differ, depending on technique of measurement used (19). Most widely used approach is applanation tonometry (SphygmoCor device), approach is historically older and uses carotid or radial pulses for pressure wave sensing and in the next step it is necessary to calibrate this wave using brachial BP measurement. Newer and simpler methods use the oscillometry technique, where the pressure wave is detected by blood pressure cuff on arm over the brachial artery or radial artery $(3,20)$. These devices are easy to use and are operator independent, which enables using such devices by clinicians in routine clinical work (5).

Based on CV risk prevalence among hypertensives and healthy persons, the reference values for cSBP were suggested. European working group had suggested the sex specific cut-off values of CSBP for diagnosis of hypertension (cSBP $\geq 133 \mathrm{mmHg}$ for males and cSBP $\geq 137 \mathrm{mmHg}$ for females) published in European Heart Journal in 2014 (21). American working group (in 2013) suggested the cut-off value of cSBP for hypertension $\geq 130 / 90$ $\mathrm{mmHg}$; the reference value for optimal $<110 / 80$ and prehypertension 110-129/80-89 mmHg (22). Similar cut-off value (112 $\mathrm{mmHg}$ ) for optimal central blood pressure was suggested on the basis of major adverse cardiovascular events occurrence during almost 9-year follow-up (23).

Many papers refer that cSBP is more strongly associated with target organ damage than brachial blood pressure $(2,5,22,24)$, which emphasizes the necessity of measuring the cSBP. When using brachial BP only for the evaluation of therapeutic efficacy of current patient antihypertensive medication, we may omit the central systolic hypertension, which has deleterious effect on heart, brain, kidneys and arteries. Brachial blood pressure may overestimate the control of hypertension $(25,26)$. Patients may suffer from unrecognized hypertension with all negative consequences. This unrecognised central hypertension could be the part of a residual risk, which is an unresolved problem in hypertension treatment (25).

For the follow-up of patients with hypertension, using both central and brachial BP measurement is necessary: not only for the evaluation of the difference between cSBP and brachSBP, but also because both values of blood pressure bring a complementary information important for managing hypertension (27). Prevailing central systolic or brachial systolic BP in individual patient can be expressed also by cSBP/brachSBP ratio; the value above 1.0 expresses a higher central systolic BP; value below 1.0 expresses a higher brachSBP. The prevalence of central systolic hypertension is very frequent in isolated systolic hypertension (ISH) and is frequently associated with a central obesity (26).

Our results showed that the prevalence of higher central systolic over brachial systolic blood pressure values was approximately $55-60 \%$ among the hypertensive patients $(7,11)$, with a significantly high repeatability of the type of central hemodynamics in the patients with treated hypertension.

The higher cSBP than brachSBP may be the part of a residual risk in treated hypertensive patients and from this aspect it needs further research $(25,28)$.

Evaluation and follow-up of both brachial BP and central BP, and also the difference between cSBP - brachSBP (or ratio), in every individual patient, should be incorporated as a promising parameter in personalized approach in treatment of hypertension.

\section{Learning points}

- Central systolic blood pressure is more strongly related to target organ damage than brachial BP.

- Higher central systolic blood pressure (cSBP) than brachial systolic blood pressure (brachSBP) have 55-60\% of treated hypertensive patients.

- Higher central than brachial systolic blood pressure increases the cardiovascular risk.

- Residual CV risk in treated hypertensive patients may partially be explained by a higher central systolic blood pressure than Brachial systolic blood pressure.

\section{Conclusions}

1. We examined the central systolic blood pressures and brachial systolic blood pressures by non-invasive oscillometric device (Arteriograph) in 83 treated hypertensive patients.

2. We found out that $60 \%$ of them had a higher central systolic blood pressure than the brachial systolic blood pressure, which was surprisingly high proportion, in contrary to traditional view. Traditional opinion states that brachial blood pressure is always higher than central blood pressure.

3. Repeated measurements in the interval of several months to years revealed the similar results. Pearson Chi-squared test of 
independence proved a statistically significant association between 1 st and 2 nd measurements.

4. For simplifying these results, we compared the central to brachial systolic blood pressure ratios; we found no significant differences between the ratios obtained in first measurement and in second measurement.

5. Statistical analysis using non-parametric Paired Wilcoxon test revealed value of $p=0.785$. According to newer opinion, a higher central systolic blood pressure than brachial blood pressure is a marker of increased cardiovascular risk and may help to elucidate the residual cardiovascular risk in patients with arterial hypertension.

\section{References}

1. McEniery CM, Cockcroft JR, Roman MJ et al. Central blood pressure: current evidence and clinical importance. Eur Heart J 2014; 35 (26): 1719-1725.

2. Kollias A, Lagou S, Maria Elena Zeniodi ME et al. Association of Central Versus Brachial Blood Pressure With Target-Organ Damage. Systematic Review and Meta-Analysis. Hypertension 2016; 67: 183-190. DOI: 10.1161/ HYPERTENSIONAHA.115.06066.

3. Jatoi NA, Mahmud A, Bennett KK, Feely J. Assessment of arterial stiffness in hypertension: comparison of oscillometric (Arteriograph), piezoelectronic (Complior) and tonometric (SphygmoCor) techniques. J Hypertens 2009; 27: 2186-2191.

4. Horváth IG, Németh $A$, Lenkey $Z$ et al. Invasive validation of a new oscillometric device (Arteriograph) for measuring augmentation index, central blood pressure and aortic pulse wave velocity. J Hypertens 2010; 28 (10): 2068-2075.

5. Wilkinson IB, McEniery CM. Cockcroft JR. Central blood pressure estimation for the masses moves a step closer. Commentary. J Human Hypertens 2010; 24: 495-497.

6. London GM, Pannier B, Safar M. Arterial stiffness, systemic reflection coefficient, and pulsatile pressure wave transmission in essential hypertension. Hypertension 2019; 74: 1366-1372. DOI: 10.1161/HypertensionAHA.119.13387.

7. Bulas J, Potočárová $\mathbf{M}$, Kupčová $\mathbf{V}$ et al. Central systolic blood pressure increases with aortic stiffness. Bratisl Med J 2019; 120 (12): 894-898.

8. Chemla D, Millasseau S. A systematic review of invasive, high-fidelity studies documenting the amplification of blood pressure from the aorta to brachial and radial arteries. J Clin Monit Comp 2020; published online 09 0ct, 2020. https: //doi.org/10.1007/s10877-020-00599-4

9. Kim H, Kim In-Cheol, Hwang J et al. Features and implicatons of higher systolic central than peripheral blood pressure in patient at very high risk of atherosclerotic cardiovascular disease. J Human Hypertens 2021; published online 06 January 2021, doi.org/10.1038/s41371-020-00472-6.

10. Ryuzaki M, Morimoto S, Niiyama $M$ et al. The relationships between differences in Central Blood Pressure and Brachial Blood Pressure and other factors in patients with essential Hypertension. Intern Med 2017; 56: 587-596.

11. Bulas, J, Potočárová M, Murín $\mathbf{J}$ et al. Central systolic hypertension in patients with well-controlled hypertension. Biomed Res International, Vol 2017, Article ID 8158974,10 pages; 2017/DOI: https//doi. org/10.1155/8158974.

12. Díaz A, Bia D. Association between central-peripheral blood pressure amplification and structural and functional cardiac properties in children, Adolescents, and Adults: Impact of the amplification parameter, recording system and calibration scheme. High Blood Press Cardiovasc Prevent 2021; 28: $185-249$.

13. Pelazza BB, Sladanha-Rosa CA, Ferreira Filho SR. Comparison between the central and brachial blood pressure values in patients with hypertension undergoing cineangiography. J Bras Nefrol 2012; 34 (3): 266-271.

14. McEnieri CM, McDonnell YB, Munnery $M$ et al. On Behalf of the Anglo-Cardiff Collaborative Trial Investigators. Central pressure: variability and impact of cardiovascular risk factors. The Anglo-Cardiff Collaborative Trial II. Hypertension 2008; 51: 1476-1482.

15. Townsend RR Wilkinson IB, Schiffrin EL et al, on behalf of the American Heart Association Council on Hypertension. Recommendations for Improving and Standardizing Vascular Research on Arterial Stiffness. A Scientific Statement from the American Heart Association. Hypertension 2015; 66: 698-722.

16. Arteriograph user's manual: http: //www.tensiomed.com/en/productarteriograph-en.

17. Nichols WW, O'Rourke MF, Vlachopoulous C. Mc Donald's blood flow in arteries. Theoretical, experimental and clinical principles," 6th Ed, Hodder Arnold, London 2011. 755 pp.

18. Boutourie P, Chowienczyk P, Humphrey JD, Mitchell GF. Arterial Stiffness and cardiovascular risk in Hypertension. Circulation Res 2021; 128 : 864-886. DOI: 10.1161/CIRCRESAHA,121.121.31861.

19. Benas D, Kornelakis M, Triantafylidi $H$ et al. Pulse wave analysis using the Mobil-O-Graph, Arteriograph and Complior device: a comparative study. Blood Pressure, 2019; 28 (2): 107-113. DOI: 10.1080/08037051.2018.1564236.

20. Trinkmann F, Benck Urs, Halder J et al. Automated noninvasive central blood pressure measurements by oscillometric radial pulse wave analysis: Results of the MEASURE-cBP validation studies. Am. J Hypertens, Ltd 2020. DOI: $10.1093 /$ ajh/174.

21. Herbert A, Cruickshank JK, Laurent S, Boutouyrie P. on behalf of The Reference Values for Arterial Measurements Collaboration: "Establishing reference values for central blood pressure and its amplification in a general healthy population and according to cardiovascular risk factors". Eur Heart J 2014; 35: 3122-3132.

22. Cheng HM, Chuang SY, Sung SH, Yu WC et al. Derivation and validation of diagnostic thresholds for central blood pressure measurements based on long-term cardiovascular risks. JACC 2013; 62 (19): 1780-1787.

23. Lamarche F, Agharazi M, Madore F, Goupil R. Prediction of cardiovascular events by type I central systolic blood pressure. A prospective study. Hypertesion 2021; 77: 319-327. DOI: 10.1161/HYPERTENSIONAHA. 120.16163 .

24. Litwin M, Obrycki L, Niemirska A et al. Central systolic blood pressure and central pulse pressure predict left ventricular hypertrophy in hypertensive children. Pediatric Nephrol 2019; 34: 703-712.

25. Baba BS, Johan PT, Mohan JC. Comparison of central aortic pressure to brachial artery presssure in hypertensive patients on drug treatment. An observational study. Indian Heart J 2019; 70: S208-S212.

26. Chuang SY, Chang HY, Tsai TY et al. Isolated systolic hypertension and central blood presssure: Implications from the national nutrition and health survey in Taiwan. J Clin Hypertens 2021; 23: 656-664.

27. Yu Shikai, Xiong Jing, Lu Yuyan et al. The prevalence of central hypertension defined by a central blood pressure type I device and its association with target organ damage in the community-dwelling elderly Chinese: The Northern Shanghai Study. J Amer Soc Hypertens 2018; 12 (3): 211-219.

28. Rinaldi ER, Yannoutsos A, Borgji $C$ et al. Central hemodynamics for risk reduction strategies: Additive value over and above brachial blood pressure. Curr Pharm Design 2015; 21: 730-736.

Received March 25, 2021. Accepted March 29, 2021. 